\title{
Impact of the Lok-bar for High-precision Radiotherapy with Tomotherapy
}

\author{
MAKOTO HIRATA ${ }^{1}$, HAJIME MONZEN ${ }^{1}$, MIKOTO TAMURA ${ }^{1,2}$, KAZUKI KUBO $^{1,3}$, KENJI MATSUMOTO $^{1,2}$, \\ KOHEI HANAOKA ${ }^{1,2}$, MASAHIKO OKUMURA ${ }^{2}$ and YASUMASA NISHIMURA ${ }^{4}$ \\ ${ }^{1}$ Department of Medical Physics, Graduate School of Medical Science, Kindai University, Osaka, Japan; \\ ${ }^{2}$ Department of Central Radiology, Kindai University Hospital, Osaka, Japan; \\ ${ }^{3}$ Department of Radiation Oncology, Tane General Hospital, Osaka, Japan; \\ ${ }^{4}$ Department of Radiation Oncology, Faculty of Medicine, Kindai University, Osaka, Japan
}

\begin{abstract}
Background/Aim: Patient immobilization systems are used to establish a reproducible patient position relative to the couch. In this study, the impact of conventional lokbars for CT-simulation (CIVCO-bar) and treatment (iBEAMbar) were compared with a novel lok-bar (mHM-bar) in tomotherapy. Materials and Methods: Verification was obtained as follows: $i$. artifacts in CT images; ii. dose attenuation rate of lok-bar, compared to without lok-bar; and iii. dose differences between the calculated and measured absorbed doses. Results: With the CIVCO-bar, there were obvious metal artifacts, while there were nearly no artifacts with the mHM-bar. The mean dose attenuation rates with the mHM-bar and $i B E A M$-bar were $1.31 \%$ and $2.28 \%$, and the mean dose difference was $1.55 \%$ and $1.66 \%$ for $\mathrm{mHM}$-bar and iBEAM-bar. Conclusion: Using the $\mathrm{mHM}$-bar reduced artifacts on the CT image and improved dose attenuation are obtained. The lok-bar needs to be inserted as a structure set in treatment planning with tomotherapy.
\end{abstract}

Indexed patient immobilization systems are commonly used to establish a reproducible patient position relative to the couch for employing high-precision radiotherapy techniques (1). However, treatment beams are then delivered through the couch top and patient immobilization system. This results in potential dosimetric effects, including increased skin dose, reduced tumor dose and altered dose distribution (2), which may have clinically significant effects (3). Low-radiationabsorbent devices are required to decrease dosimetric impact. Monzen et al. developed a lok-bar (HM-bar) used to secure

Correspondence to: Hajime Monzen, Ph.D., Department of Medical Physics Graduate School of Medical Science, Kindai University, 3772 Ohno-Higashi, Osakasayama, 589-8511 Osaka, Japan. Tel: +81 723660221, Fax: +81 723660206, e-mail: hmon@med.kindai.ac.jp

Key Words: Artifacts, immobilizer, lok-bar, tomotherapy. the immobilizers to the treatment couch, finding that the HM-bar, compared with a conventional lok-bar, improved the dose attenuation rate in stereotactic body radiation therapy (SBRT), using volumetric modulated arc therapy with a linear accelerator (Linac) for lung cancer (4). The helical beams of tomotherapy are irradiated continuously from whole gantry angles (5). The frequency of the beam passing through the lok-bar is higher than in the Linac, which may significantly impact dosimetry. However, impacts of the lok-bar on dose calculation and attenuation have not been delineated. In addition, the previous study on tomotherapy showed that there was no difference between measured and calculated absorbed doses in the irradiation field without the lok-bar (6). Lok-bars may also be a factor influencing uncertainty.

In this study, conventional lok-bars for CT-simulation and treatment were compared with a modified novel lowradiation-absorbent lok-bar, based on: 1) artifacts caused by each lok-bar in CT images; 2) the dose attenuation rate of each lok-bar versus the absorbed dose without the lok-bar, in 9 delivery quality assurance (DQA) plans for lung, head, head-and-neck, abdominal and pelvic cancers; and 3) the differences between calculated and measured absorbed doses in the 9 DQA plans.

\section{Materials and Methods}

Specifications and components of each lok-bar: CIVCO-bar, $i B E A M$-bar and $m H M$-bar. Figure 1 shows the mHM-bar (Nichigen Co. Ltd, Tokyo, Japan) (Figure 1A lower, B and C) a modification of the HM-bar to adjust the fixation to fit the various sizes of the couch. Also shown are conventional lok-bars for treatment (iBEAMbar, iBEAM-bar ${ }^{\circledR}$ evo CT Overlay, Elekta Oncology Systems, Crawley, UK) (Figure 1A middle and D) and CT-simulation (CIVCO-bar) (Figure 1A upper and E).

The mHM-bar was constructed from a carbon fiber-reinforced plastic bar and the pin was polyacetal resin blocks (4). The iBEAMbar and CIVCO-bar were constructed from metal materials. The CT couch was $53 \mathrm{~cm}$ wide and the treatment couch was $50 \mathrm{~cm}$ wide. 

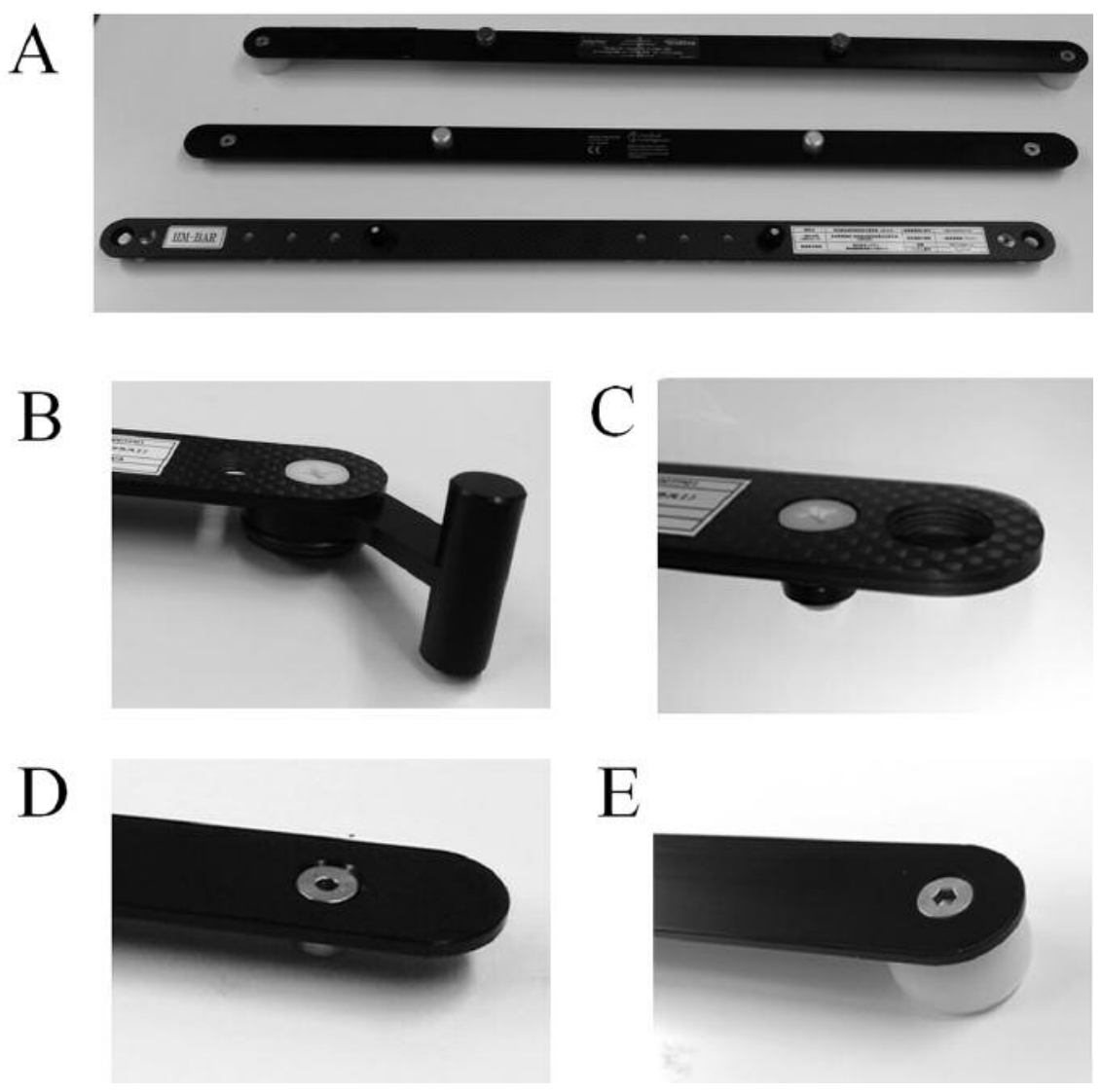

Figure 1. Photographs showing the CIVCO-bar, iBEAM-bar and mHM-bar. (A) CIVCO-bar (upper), iBEAM-bar (middle) and mHM-bar (lower); (B) The component of the mHM-bar that is affixed to the CT couch; $(C)$ The component of the mHM-bar that is affixed to the tomotherapy couch; (D) The component of the iBEAM-bar that is affixed to the tomotherapy couch; $(E)$ The component of the CIVCO-bar that is affixed to the CT couch. The new version features two extra knobs on the two sides allowing attachment for universal compatibility with CT-simulation and the treatment couch. It also offers 8 knobs in the middle of the bar, allowing for adjustability in all types of immobilizers.

Artifacts in CT images: CIVCO-bar vs. mHM-bar. The CIVCO-bar and $\mathrm{mHM}$-bar were positioned on the couch and the center of the I'mRT phantom (IBA Dosimetry GmbH, Schwarzenbruck, Germany) and scanned using a 16-slice CT scanner (Toshiba Aquilion LB, Toshiba Medical Systems Co., Tochigi, Japan). The imaging conditions were set to tube voltage $120 \mathrm{kV}$, tube current $400 \mathrm{~mA}$, slice thickness $2.0 \mathrm{~mm}$ and scan range $\pm 200 \mathrm{~mm}$ from the center of the phantom and lok-bar. The field of view (FOV) was $598.8 \mathrm{~mm}$.

An acrylic plate was placed on the lok-bars to secure them to the phantom. Images were scanned without the lok-bars (non-bar), or with the CIVCO-bar or mHM-bar. The subtracted CT images were obtained, each as the CT image with the CIVCO-bar or mHM-bar minus the CT image with the non-bar, determined by image analysis with Image J software (National Institutes of Health, Bethesda, MD, USA). Then, the subtracted CT images were compared to visually evaluate the artifacts. In addition, profile curves were drawn for each subtracted CT image in the $\mathrm{X}$-axis.

Dose attenuation and dose difference between calculated and measured absorbed doses in the DQA plans: iBEAM-bar vs. $m H M$ bar. The dose attenuation and dose difference between calculated and measured absorbed dose were investigated for each lok-bar in tomotherapy (Accuray Inc., Madison, WI, USA). First, we created DQA plans with RTPS (Planning Station, 5.0.5.18 19-Nov-2014: Accuray Inc., Madison, WI, USA) using CT images obtained as described above (in Artifacts in CT images: CIVCO-bar vs. mHMbar section). In these CT images, the part of the planning CT couch under the surface was replaced with a treatment digital couch (5). In this study, 9 DQA plans were evaluated, as shown in Table I. The $\mathrm{x}$-ray energy was $6 \mathrm{MV}$ and a mini ionization chamber (CC01, Wellhofer-Scanditronix, Bartlett, TN, USA) was set at the center of the I'mRT phantom to determine the dose attenuation rate for each lok-bar. The center of the I'mRT phantom corresponded to the center of the Planning Target Volume (PTV) for each clinical radiation treatment site. The absorbed doses for each bar were calculated by the RTPS and measured with the mini ionization chamber. Measurements were performed 3 times for each DQA plan.

The calculated and measured absorbed doses with the non-bar were defined as $100 \%$ and the attenuation rates of the dose for the calculated and measured absorbed doses for each bar were determined using equation [1]. In addition, the dose difference between calculated and measured absorbed doses for each lok-bar were determined using equation [2]. 
Table I. Summary of the 9 helical tomotherapy plans, including couch travel, irradiation time, jaw width and total prescription dose.

\begin{tabular}{lccccc}
\hline Plan number & Target Organ & $\begin{array}{c}\text { Couch travel } \\
(\mathrm{cm})\end{array}$ & $\begin{array}{c}\text { Irradiation time } \\
(\mathrm{sec})\end{array}$ & $\begin{array}{c}\text { Jaws width } \\
(\mathrm{cm})\end{array}$ & $\begin{array}{c}\text { Total prescription } \\
\text { dose(Gy)/fraction }\end{array}$ \\
\hline 1 & Lung (origin) & 7.6 & 460.7 & 2.5 & $50 / 5$ \\
2 & Whole Neck (Oropharyngeal cancer) & 22.0 & 240.4 & 2.5 & $70 / 35$ \\
3 & Prostate & 9.0 & 172.5 & 2.5 & $74 / 37$ \\
4 & Brain (metastasis) & 6.5 & 225.4 & 2.5 & $40 / 5$ \\
5 & Lung (origin) & 5.8 & 444.5 & 2.5 & $60 / 8$ \\
6 & Brain (metastasis) & 4.6 & 177.4 & 1.0 & $40 / 5$ \\
7 & Pelvis (Lympho Node) & 5.2 & 193.2 & 2.5 & $54 / 30$ \\
8 & Adrenal (Lymphoma) & 13.6 & 161.4 & & $40 / 20$ \\
9 & Prostate & 7.9 & 128.9 & & 2.5 \\
\hline
\end{tabular}

Attenuation rate $=\frac{\left.\begin{array}{c}(\text { Calculated and measured dose } \\ \text { without } \text { lok }_{\text {bar }} \text {-calculated and } \\ \text { measured dose } \text { with }_{\text {lok }} \text { bar }\end{array}\right)}{\begin{array}{c}\text { Calculated and measured } \\ \text { dose without lok }\end{array}}$

Dose difference $=\frac{\begin{array}{c}\left(\text { Calculated dose with } \text { lok }_{\text {bar }}-\right. \\ \left.\text { measured dose with lok }{ }_{\text {bar }}\right)\end{array}}{\text { Calculated dose with lok } \text { lor }_{\text {bar }}}$

The Wilcoxson ranked test was used to compare the differences between the calculated and measured doses for each lok-bar. Statistical analyses were performed with EZR on R Commander, version 3.3.2 (Saitama Medical Center, Jichi Medical University, Saitama, Japan) (7) and $p<0.05$ was considered statistically significant.

\section{Results}

Artifacts in CT images: CIVCO-bar vs. mHM-bar. Figure 2 shows CT images of the axial plane of the centers of the CIVCO-bar and mHM-bar. In the CIVCO-bar, the metal artifacts from two pins and the component used to stabilize the couch were readily detectable (Figure 2A). However, there were almost no artifacts with the mHM-bar (Figure 2B). In the CT value profiles (Hounsfield Units (HU)) for the axial plane of the CIVCO-bar, the mean, maximum, minimum and standard deviation (1SD) were 47.34, $322.00,-49.00$ and $45.38 \mathrm{HU}$, respectively (Figure 2E). For the mHM-bar, the mean, maximum, minimum and standard deviation (1SD) were 11.59, 56.00, -44.00 , and 16.34 HU, respectively (Figure $2 \mathrm{~F}$ ).

Dose attenuation and dose differences between calculated and measured absorbed doses in DQA plans: iBEAM-bar vs.
mHM-bar. Table II shows the attenuation rates of calculated and measured absorbed doses for each lok-bar, compared with the non-bar, in 9 DQA plans. Figure 3 shows the measured relative doses for each bar normalized by the measured absorbed dose for the non-bar. The mean dose attenuation rate of calculated absorbed doses for the mHMbar and CIVCO-bar was $1.15 \%$ and $2.37 \%$, respectively. The mean dose attenuation rate of measured absorbed doses for the mHM-bar and iBEAM-bar was $1.31 \%$ and $2.28 \%$, respectively.

Table III shows differences between the calculated and measured absorbed doses for each lok-bar in the 9 DQA plans. The mean dose difference for the mHM-bar and iBEAM-bar was $1.55 \%$ and $1.66 \%$, respectively. The maximum dose difference for the mHM-bar and iBEAM-bar was $2.28 \%$ and $2.55 \%$, respectively. The dose differences of the mHM-bar and iBEAM-bar were not significantly different $(p=0.426)$.

\section{Discussion}

In this study, the artifacts in CT images, impacts of these artifacts on dose calculation, dose attenuation rates and differences between calculated and measured absolute doses for each lok-bar in tomotherapy were investigated.

In general, metal artifacts may affect dose calculations (3). In some cases, treatment planning is performed with metal artifacts from the CIVCO-bar in CT images, with examples shown in Figure 2A and $\mathrm{C}$. However, the mHM-bar generated very few artifacts compared with the CIVCO-bar, as shown in Figure 2C and D. In other words, use of the mHM-bar could minimize the problems affecting the dose calculation, accuracy of target contour and precision of image registration in high-precision radiotherapy with tomotherapy. The averages of the CT-value profiles were 47.34 HU and 11.59 HU for the CIVCO-bar and mHM-bar, respectively. The difference in $\mathrm{CT}$ values between the 

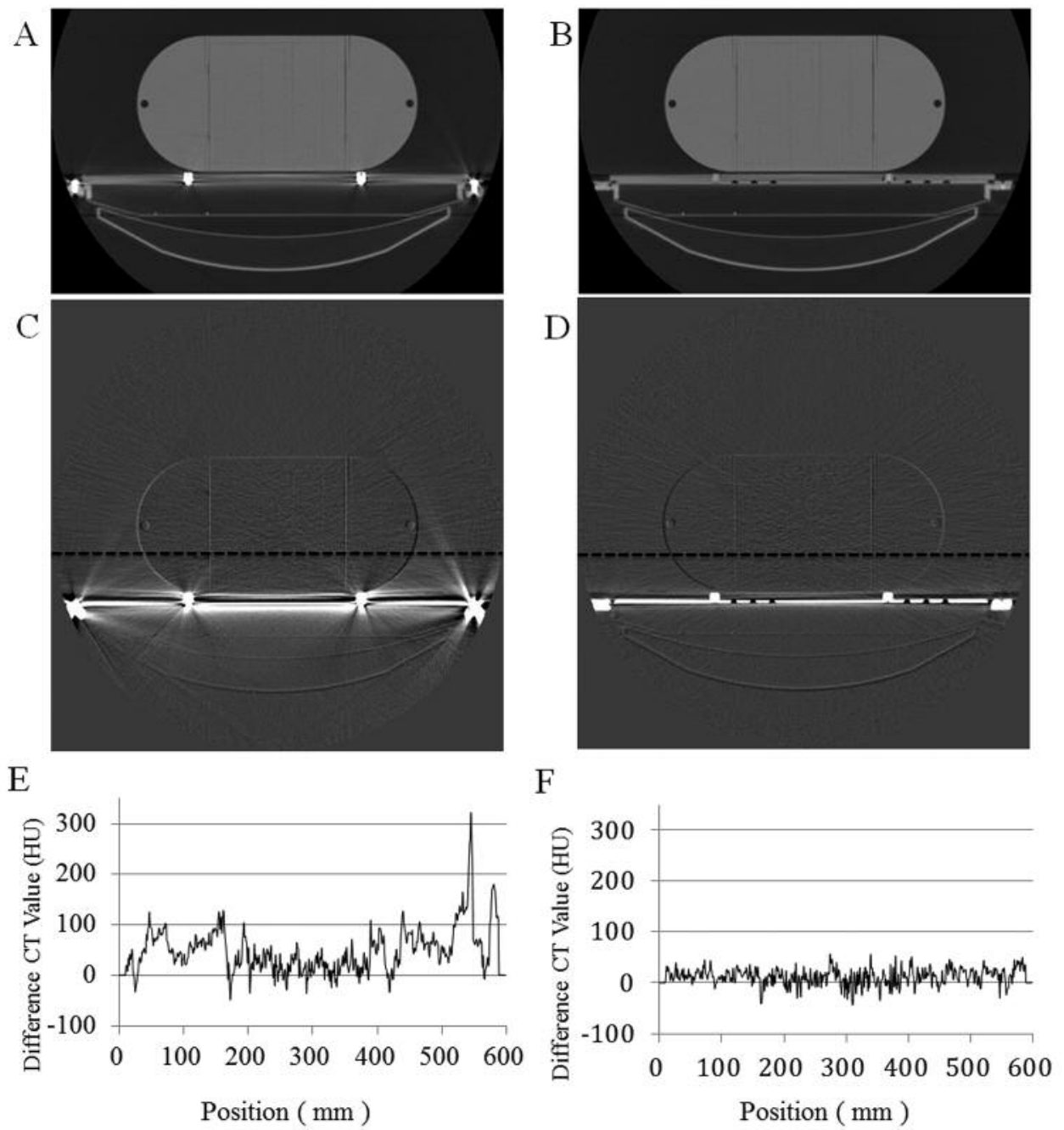

Figure 2. Artifacts produced by the lok-bars. (A) CIVCO-bar; (B) mHM-bar; (C) CIVCO-bar, subtracted CT image; (D) mHM-bar, subtracted CT image; (E) Profile curve for the CT values regarding the subtracted image of the CIVCO-bar [black dotted line in $(C)] ;(F)$ Profile curve for the $C T$ values regarding the subtracted image of the mHM-bar [black dotted line in (D)]. The part of the planning CT couch under the surface was not calculated because it was replaced with the digital couch for the calculation.

Table II. Attenuation rates between calculated/measured dose with the non-bar and calculated/measured doses with each lok-bar, for 9 DQA plans. The attenuation rate was calculated using Equation 2.

\begin{tabular}{|c|c|c|c|c|}
\hline \multirow[t]{2}{*}{ Plan number } & \multicolumn{2}{|c|}{ Attenuation rates of calculated dose (\%) } & \multicolumn{2}{|c|}{ Attenuation rates of measured dose $(\%)$} \\
\hline & non-bar/mHM-bar & non-bar/CIVCO-bar & non-bar/mHM-bar & non-bar/iBEAM-bar \\
\hline 1 & 1.15 & 1.97 & 1.20 & 2.30 \\
\hline 2 & 1.04 & 1.71 & 1.37 & 2.38 \\
\hline 3 & 0.80 & 2.16 & 1.55 & 2.61 \\
\hline 4 & 1.04 & 2.06 & 1.19 & 2.63 \\
\hline 5 & 1.24 & 2.12 & 1.71 & 2.76 \\
\hline 6 & 1.27 & 1.48 & 1.78 & 2.37 \\
\hline 7 & 1.18 & 5.49 & 1.06 & 2.13 \\
\hline 8 & 1.07 & 1.93 & 0.80 & 1.47 \\
\hline 9 & 1.60 & 2.38 & 1.10 & 1.87 \\
\hline Average & 1.15 & 2.37 & 1.31 & 2.28 \\
\hline
\end{tabular}


Table III. Influence of two lok-bars on radiation dose differences between calculated and measured absorbed dose in the 9 DQA plans.

\begin{tabular}{|c|c|c|c|c|c|c|c|c|}
\hline \multirow[t]{2}{*}{ Plan number } & \multicolumn{4}{|c|}{ mHM-bar } & \multicolumn{4}{|c|}{ iBEAM-bar } \\
\hline & $\begin{array}{c}\text { Calculated } \\
\text { dose (Gy) }\end{array}$ & $\begin{array}{l}\text { Measured } \\
\text { dose (Gy) }\end{array}$ & $\begin{array}{l}\text { Standard } \\
\text { Deviation }\end{array}$ & $\begin{array}{c}\text { Dose } \\
\text { difference }(\%)\end{array}$ & $\begin{array}{c}\text { Calculated } \\
\text { dose (Gy) }\end{array}$ & $\begin{array}{l}\text { Measured } \\
\text { dose (Gy) }\end{array}$ & $\begin{array}{c}\text { Standard } \\
\text { Deviation }\end{array}$ & $\begin{array}{c}\text { Dose } \\
\text { difference }(\%)\end{array}$ \\
\hline 1 & 8.812 & 8.652 & 0.001 & 1.81 & 8.739 & 8.555 & 0.005 & 2.10 \\
\hline 2 & 1.652 & 1.643 & 0.000 & 0.59 & 1.641 & 1.626 & 0.001 & 0.94 \\
\hline 3 & 1.959 & 1.916 & 0.001 & 2.22 & 1.932 & 1.895 & 0.001 & 1.92 \\
\hline 4 & 6.680 & 6.538 & 0.000 & 2.13 & 6.611 & 6.442 & 0.004 & 2.55 \\
\hline 5 & 6.907 & 6.749 & 0.005 & 2.28 & 6.845 & 6.677 & 0.006 & 2.45 \\
\hline 6 & 6.880 & 6.750 & 0.084 & 1.88 & 6.865 & 6.709 & 0.002 & 2.27 \\
\hline 7 & 1.684 & 1.651 & 0.000 & 1.96 & 1.611 & 1.633 & 0.001 & 1.40 \\
\hline 8 & 1.747 & 1.751 & 0.002 & 0.21 & 1.732 & 1.739 & 0.001 & 0.41 \\
\hline 9 & 1.903 & 1.886 & 0.006 & 0.87 & 1.888 & 1.872 & 0.001 & 0.85 \\
\hline Average (\%) & & & & 1.55 & & & & 1.66 \\
\hline Standard Deviation & & & & 0.78 & & & & 0.78 \\
\hline
\end{tabular}

CIVCO-bar and mHM-bar was approximately $35 \mathrm{HU}$. We found that calculated doses for the mHM-bar were higher than those for the CIVCO-bar in all cases, as shown in Table II. This was caused by differences in CT values and might also indicate that artifacts in the CT image affected the dose calculations.

The mean dose difference of the calculated doses for the mHM-bar and the CIVCO-bar was $1.15 \%$ and $2.37 \%$, respectively, in 9 DQA plans while the mean dose difference of the measured doses was $1.31 \%$ and $2.28 \%$, respectively (Table II). In addition, the mean differences between calculated and measured absorbed doses were greater than $1.0 \%$ for all the lok-bars shown in Table III. Especially, in the case of using a slow couch speed in the treatment (5) such as the plan numbers of $1,4,5,6$ and 7, there was more likely a large difference between the calculated and measured doses, compared with the other cases. The couch and lok-bar in the irradiation field might have caused the large dose differences between the calculated and measured doses in the case where the couch speed was slow, because the shielding effect of the lok-bar was large attributable to the helical tomotherapy. In the dose calculation with RTPS of tomotherapy, the pin of the lok-bar or planning CT couch was excluded because the part of planning CT couch under the surface was replaced with the treatment digital couch (5). Therefore, the difference between the calculated and measured absolute doses was more than $1.0 \%$ for all lokbars. This indicated that a difference between the calculated and measured delivery dose to a patient might occur when the bar is inside the irradiation field. The dose error between calculated and measured absorbed doses, approximately $1 \%$ for all lok-bars, is a level that cannot be ignored in clinical applications. In this study, the metal artifact in CT image

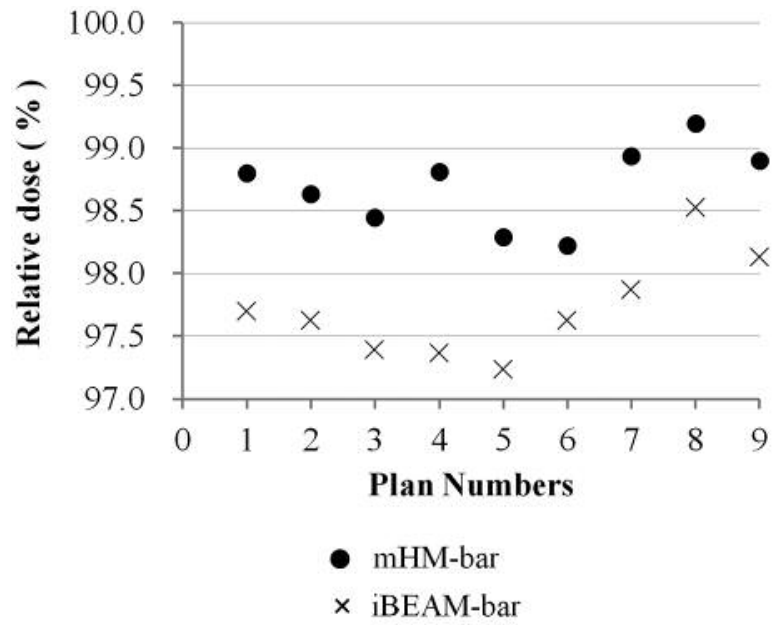

Figure 3. Relative measured doses for each lok-bar, each normalized by the measured dose with the non-bar.

with CIVCO-bar did not impact the dose error since the convolution/superposition method was used for the dose calculation engine (5). In this dose calculation engine, the CT number to electron density conversion factor may not account for the imaging artifact (8). On the other hand, in the most advanced dose calculation algorithm such as Acuros XB (Varian Medical Systems, Palo Alto, CA, USA) and X-ray Voxel Monte Carlo (BrainLAB iPlan, Feldkirchen, Germany), the CT numbers for each voxel are converted to material mapping which defines material type and mass density (9). Therefore, the metal artifact might have more impact on the dose error by using those algorithms in high precision radiotherapy with tomotherapy. 
Monzen et al. showed that the reduction rates of the absolute dose for SBRT with a Linac were $1.0 \%$ and $1.9 \%$ for the conventional-bar and an HM-bar, respectively (4). The reduction rate in this study was higher than that in the previous study using a treatment other than SBRT. The impact of the lok-bar for tomotherapy was higher than that for the Linac because the helical beams of tomotherapy were irradiated continuously from whole angles. Paula et al. described tomotherapy could improve the ability to adhere to dose-constraint parameter for IMRT (10). The influence of the lok-bar must be investigated since the positional precision and reproduction by use of immobilization devices are required in IMRT. It may apply to newly developed devices, such as Halcyon (Varian Medical Systems, Palo Alto, CA, USA) and Radixact (Accuray Inc., Sunnyvale, CA, USA). From this standpoint, the improvement in dose variation by using the mHM-bar, was a significant achievement.

In some studies, it was reported that, from a theoretical radiobiology perspective, the dose variation should be $3 \%$ of the prescribed radiation dose to maximize efficacy of the radiation therapy (11-13). Many factors can affect the dose variation, including the immobilization device. This study also suggested that the lok-bar accounted for more than $1.0 \%$ of the dose variation. Therefore, the lok-bar should be considered in calculations as a structure set in treatment planning for high-precision radiotherapy with tomotherapy.

\section{Conclusion}

Using the mHM-bar could reduce artifacts in CT-simulation and improve dose attenuation. The index-bar needs to be inserted as a structure set in treatment planning for highprecision radiotherapy with tomotherapy.

\section{Conflicts of Interest}

Hajime Monzen has a consultancy agreement with, and financial interest in, Toppan Printing Co., Ltd., Tokyo, Japan.

\section{Acknowledgements}

This work was supported by the JSPS KAKENHI grant numbers 17K09071 and 16K09027. The Authors thank Mr. Masaru Hayakawa for his valuable support.

\section{References}

1 Cheng KF and Wu VWC: Comparison of the effectiveness of different immobilization systems in different body regions using daily megavoltage $\mathrm{CT}$ in helical tomotherapy. $\mathrm{Br} \mathrm{J}$ Radiol 87(1034): 20130494, 2014.

2 Jeraj R, Mackie TR, Balog J, Olivera G, Pearson D, Kapatoes J, Ruchala K and Reckwerdt P: Radiation characteristics of helical tomotherapy. Med Phys 31: 396-404, 2004.

3 Olch AJ, Gerig L, Li H, Mihaylov I and Morgan A: Dosimetric effects caused by couch tops and immobilization devices: Report of AAPM Task Group 176. Med Phys 41: 061501, 2014.

4 Monzen H, Kubo K, Tamura M, Hayakawa M and Nishimura Y: Development of a novel low-radiation-absorbent lok-bar to reduce $\mathrm{X}$-ray scattering and absorption in RapidArc ${ }^{\circledR}$ treatment planning and dose delivery. J Appl Clin Med Phys 18: 44-51, 2017.

5 Langen KM, Papanikolaou N, Balog J, Crilly R, Followill D, Goddu SM, Grant W, Olivera G, Ramsey CR and Shi C: QA for helical tomotherapy: Report of the AAPM Task Group 148. Med Phys 37: 4817-4853, 2010.

6 Hirata M, Monzen H, Hanaoka $\mathrm{K}$ and Nishimura Y: Measurement of absorption dose outside irradiation field in IMRT. Radiation protection dosimetry. 176: 425-433, 2017.

7 Kanda Y: Investigation of the freely available easy-to-use software 'EZR' for medical statistics. Bone Marrow Transplant 48: 452-458, 2013.

8 Reft C, Alecu R, Das IJ, Gerbi BJ, Keall P, Lief E, Mijnheer BJ, Papanikolaou N, Sibata C and Van Dyk J: Dosimetric considerations for patients with HIP prostheses undergoing pelvic irradiation: Report of the AAPM Radiation Therapy Committee Task Group 63. Med Phys 30: 1162-1182, 2003.

9 Ojala $J$ and Kapanen M: Quantification of dose differences between two versions of Acuros XB algorithm compared to Monte Carlo simulations - The effect on clinical patient treatment planning. J Appl Clin Med Phys 16: 213-225, 2015.

10 DE Paula U, D'Angelillo RM, Barbara R, Caruso C, Gomellini S, Caccavari A, Costarelli L, Scavina P, Mauri M, Santini E, Antonaci A, Cavaliere F, LA Pinta M, Loreti A and Fortunato L: Once Daily Accelerated Partial Breast Irradiation: Preliminary Results with Helical Tomotherapy(R). Anticancer Res 36: 3035-3039, 2016.

11 Bentzen SM: High-tech in radiation oncology: should there be a ceiling? Int J Radiat Oncol Biol Phys 58: 320-330, 2004.

12 Mijnheer BJ, Battermann JJ and Wambersie A: What degree of accuracy is required and can be achieved in photon and neutron therapy? Radiother Oncol 8: 237-252, 1987.

13 Brahme A: Dosimetric precision requirements in radiation therapy. Acta Radiol Oncol 23: 379-391, 1984.

Received March 5, 2018

Revised March 25, 2018

Accepted March 26, 2018 